Meta

Journal des traducteurs

Translators' Journal

\title{
Le cerveau du traducteur
}

\section{De quelques propositions sur ce thème}

\section{Ch. P. Bouton}

Volume 29, numéro 1, mars 1984

Cerveau, langage et traduction

URI : https://id.erudit.org/iderudit/002129ar

DOI : https://doi.org/10.7202/002129ar

Aller au sommaire du numéro

Éditeur(s)

Les Presses de l'Université de Montréal

ISSN

0026-0452 (imprimé)

1492-1421 (numérique)

Découvrir la revue

Citer cet article

Bouton, C. P. (1984). Le cerveau du traducteur : de quelques propositions sur ce thème. Meta, 29(1), 44-56. https://doi.org/10.7202/002129ar

Ce document est protégé par la loi sur le droit d'auteur. L'utilisation des services d'Érudit (y compris la reproduction) est assujettie à sa politique d'utilisation que vous pouvez consulter en ligne.

https://apropos.erudit.org/fr/usagers/politique-dutilisation/
Cet article est diffusé et préservé par Érudit.

Érudit est un consortium interuniversitaire sans but lucratif composé de l’Université de Montréal, l'Université Laval et l'Université du Québec à Montréal. Il a pour mission la promotion et la valorisation de la recherche. https://www.erudit.org/fr/ 


\section{LE CERVEAU DU TRADUCTEUR DE QUELQUES PROPOSITIONS SUR CE THÈME}

CH. P. BOUTON

\section{PRÉAMBULE}

Il y aurait une certaine outrecuidance à prétendre apporter ne fût-ce que des éléments de réponse aux problèmes si complexes que suggère implicitement ce titre. L'état actuel des connaissances disponibles en ce domaine n'autorise pas même d'envisager sérieusement une démarche aussi intrépide!

D'où notre sous-titre qui souligne la nature modeste et prudente de cette entreprise. Il s'agit de rapprocher certaines données tant linguistiques que neurophysiologiques, d'examiner les concepts qui s'en sont dégagés, et dans un esprit de synthèse, de rapprocher les pièces déjà disponibles d'un jeu de patience encore bien lacunaire où l'on peut cependant deviner déjà l'esquisse d'un schéma plus cohérent.

\section{DONNÉES LINGUISTIQUES}

\section{L'ACTIVITÉ TRADUISANTE}

De nombreux auteurs ${ }^{1}$ ont contribué à définir cette activité et à en cerner les caractères spécifiques. Nous nous contentons donc de rappeler quelques distinctions nécessaires.

De la traduction spontanée, orale, de la parole de l'autre à l'effort réfléchi, sur un texte, afin d'en rendre l'équivalent dans une langue différente, il y a une infinité de situations possibles qu'enferment dans un système taxonomique assez lâche des oppositions telles que :

traduction / interprétation

traduction littérale / traduction littéraire, etc.

Mais, derrière cette diversité, une constante s'impose. Il s'agit toujours de "faire passer "un discours, au sens guillaumien du terme ${ }^{2}$, exprimé en fonction des moyens d'un code spécifique, la langue source A, en utilisant les moyens d'un autre code, ceux de la langue cible $B$. Ce " passage " est rendu possible grâce à l'intèrvention d'un médiateur, en l'occurrence un sujet parlant qui possède une compétence double : $(A+B)$, lui

1. Rappelons les travaux de

- G. Mounin (1965) : les Problèmes théoriques de la traduction, Paris, Gallimard ; (1976) : Linguistique et traduction, Bruxelles, Dressard et Mardaga.

- Ch. Taber et A. Nida (1971) : la Traduction, théories et méthodes, Londres, Alliance biblique universelle.

- U. Weinrich (1962) : Language in Contact, La Haye, 2nd. ed.

2. C'est-à-dire l'énoncé (oral) ou le texte (écrit) produit par le sujet parlant. 
permettant non seulement des performances en $\mathbf{A}$ et en $\mathbf{B}$ mais justement l'opération de passage de $\mathrm{A}$ à $\mathrm{B}$ ou l'inverse. Notons que sur le plan théorique nous occupant ici, ce médiateur devrait pouvoir être un automate, une machine.

Cette constante présuppose des variables. D'abord la nature même des performances possibles : passage de $\mathbf{A}$ à $\mathbf{B}$ ou de $\mathbf{B}$ à $\mathrm{A}$, ou indifféremment $\mathrm{A}=\mathrm{B}$. Ensuite le facteur temporel : traduction simultanée, immédiate où le facteur temporel tend vers epsilon ; traduction " à retardement " où il peut tendre vers une durée très longue. Enfin, la visée téléologique de l'activité traduisante qui peut se réaliser en fonction de différents critères : esthétique, technique, éthique, etc.

\section{LES MODÈLES LINGUISTIQUES}

Chaque fois que la réflexion linguistique se penche sur les problèmes de la traduction, elle doit lutter contre la tendance qui la conduirait à conclure que ceux-ci sont insolubles parce que l'activité traduisante elle-même est en fait théoriquement impossible. Elle doit s'incliner cependant devant l'évidence des faits. De même que l'on prouve le mouvement en marchant, l'activité traduisante a précédé de bien longtemps toute tentative de théorisation linguistique.

Pourtant, malgré les difficultés de l'entreprise, de nombreuses tentatives se sont développées depuis que la linguistique s'est constituée en science adulte, pour rendre compte, sur le plan théorique, de l'activité traduisante. Nous ne retiendrons, ici, en fonction de notre propos que les options les plus pertinentes ${ }^{3}$.

La traduction peut être envisagée d'abord comme se fondant sur l'application d'un système de règles de "transformation " 4 définissant les conditions de "réécriture " ou de "reformulation" de l'énoncé de la langue $A$, dans la langue $B$. Une longue tradition de l'enseignement des langues classiques, fondé sur l'apprentissage de tels systèmes de règles :

mirabile visu - admirable à voir

timeo ne veniat - je crains qu'il ne vienne

donne à cette démarche une garantie de rigueur. Mais le modèle ainsi proposé :

Énoncé $\mathrm{A}$ - règle $\mathrm{a}-\mathrm{b}$ - énoncé $\mathrm{B}$

réduit à une telle forme canonique ignore totalement le rôle du sujet traducteur. Il ne peut donc rendre compte de la réalité de l'opération traduisante. Celle-ci ne peut être dissociée du sujet qui en est le médiateur.

Devant l'énoncé [Ax] de la langue A, le sujet traducteur suit d'abord une première démarche, que nous appellerons démarche sémasiologique ${ }^{5}$ qui va du mot à mot du texte à son sens, ou plutôt, à l'interprétation de celui-ci par le médiateur ${ }^{6}$. Puis, il suit une démarche inverse, démarche onomasiologique, rigoureusement symétrique à la première

3. On peut consulter Ch. P. Bouton (1979) : la Linguistique appliquée, Paris, PUF, "Que sais-je ?", no 1755 , pp. $58-73$.

4. Il faut prendre, dans cette phrase, le terme transformation dans un sens moins spécifique que celui qu'on lui accorde dans la théorie de la grammaire transformationnelle et générative.

5. C'est la recherche de la signification à partir de la forme des signifiants. La recherche des signifiants à partir de l'intention de signification est la démarche inverse, la démarche onomasiologique. Voir B. Pottier (1967) : Présentation de la linguistique, Paris, Klincksieck, p. 11, et (1974) : Linguistique générale, Paris, Klincksieck, p. 30.

6. Sur le plan théorique, on doit admettre que la démarche sémasiologique aboutit, du fait de la "médiation " de celui qui écoute ou qui lit, à une re-création des intentions de dire de l'autre, celui qui a fait la démarche inverse, onomasiologique de construction de l'énoncé. Du fait de différents facteurs, dont les individus en cause, on ne saurait garantir que les valeurs signifiées au point d'origine et au point d'aboutissement sont identiques. 
qui va de ce "sens" ou son interprétation, à son expression dans un énoncé [Bx] de la langue $\mathbf{B}$.

Ce faisant, le médiateur peut puiser dans l'ensemble des règles que postule le précédent modèle. Mais cete démarche n'est pas toute l'activité traduisante, loin de là. Audelà de la mise en oeuvre de ce "savoir faire ", le médiateur fait constamment appel à un savoir qui est d'abord représenté, sur le plan linguistique par son expérience intime des deux systèmes (langue $\mathbf{A}$ et langue $B$ ) et ensuite, sur un plan extra-linguistique par son expérience directe de la réalité qui constitue la référence par rapport à laquelle se définissent les "sens" des énoncés [Ax] et [Bx'].

L'appareil dialectique emprunté aux théories de la grammaire générative et transformationnelle (nouvelles venues, relativement, dans le champ de la réflexion linguistique) permet de présenter d'une manière différente les deux modèles précédents. Comme les termes utilisés ici ont évolué, dans leur sens, avec les théories elles- mêmes, précisons que nous partirons des valeurs que leur accorde N. Chomsky dans : The Formal Nature of Language, publié en appendice à l'ouvrage de E. Lenneberg, Biological Foundations of Language, 1967, dans Cartesian Linguistics, 1966, et dans Studies on Semantics in Generative Grammar, 1972. La référence commune à ces différents textes est bien entendu : Aspects of the Theory of Syntax, qui date de 1965.

Il existe des "universaux sémantiques " comme des "universaux phonétiques" qui font partie d'une "grammaire universelle ". Celle-ci représente l'ensemble des conditions qui doivent être remplies par les grammaires de toutes les langues humaines. Elle est en fait la théorie de la structure du langage. La recherche des éléments d'une telle grammaire universelle conduit à identifier des relations constantes que l'homme établit dans sa démarche cognitive pour comprendre ${ }^{7}$ la réalité au double niveau du monde perçu et des entités pensées et senties ${ }^{8}$. Une telle recherche révèle probablement des mécanismes spécifiques du fonctionnement du cerveau humain et donc de l'élaboration des systèmes linguistiques. La probité intellectuelle nous conduit à souligner le caractère personnel de cette double réflexion.

Si l'on considère des énoncés d'une langue particulière, il faut reconnaître derrière les structures de surface qui constituent le plan de l'expression, ou du signifiant, et où s'identifient toutes les informations relevant de l'interprétation phonétique, une structure abstraite qui incorpore toutes les informations pertinentes à l'interprétation sémantique. C'est la structure profonde.

Dans Cartesian Linguistics, Chomsky rappelle, pour éclairer cette notion de structure profonde, la démarche de Lancelot et d'Arnault dans la Grammaire générale et raisonnée, 1660 . Cette structure sous-jacente, universelle, procède de la manière naturelle en laquelle nous exprimons nos pensées, suggère-t-il en citant les deux auteurs précités.

Tandis que la structure profonde sera redéfinie plus tard dans la théorie d'une façon beaucoup plus restrictive et sensiblement plus technique, elle est alors présentée ainsi :

The deep structure that expresses the meaning is common to all languages, so it is claimed being a simple reflection of the forms of thought. Cartesian Linguistics, London, New York, Harper \& Row, 1966, p. 35.

Ainsi, à ces structures profondes s'opposent dans le discours généré par chaque langue particulière des structures de surface, évidemment spécifiques à la langue considérée : où s'identifient toutes les informations relevant de l'interprétation phonétique. El-

7. Au sens étymologique du terme : se saisir

8. Le concret des choses opposé à l'abstrait des idées. 
les résultent, sur le plan de l'expression, des transformations (le terme est maintenant employé dans le sens spécifique de la théorie) des structures profondes postulées sur le plan du contenu ${ }^{9}$.

Nous n'entrerons pas dans la querelle latente de l'inné et de l'acquis où certains veulent voir dans les structures profondes un inné tandis que l'acquis se réduit à la découverte des règles de transformation qui permettent de passer de celles-ci aux structures de surface propres à telle ou telle langue. Nous nous limiterons à considérer qu'en raison de la théorie toute connaissance d'une langue se ramène d'abord à la maîtrise des règles de transformation qui permettent de passer de la structure profonde à la structure de surface particulière à cette langue et ceci aux différents niveaux où celle-ci s'organise sur le plan de l'expression : phonologie, morphologie, syntaxe, sémantique.

Compte tenu des limitations formelles imposées par la théorie de la grammaire générative et transformationnelle, le premier modèle proposé se ramène à un ensemble de règles transformationnelles qui permettent de passer des structures de surface de la langue $\mathrm{A}$ à celles de la langue $\mathrm{B}$, selon une formule :

Structure de surface [Langue A]

règles de transformation

Structure de surface [langue B]

Le second modèle intègre les deux démarches précédemment envisagées ${ }^{10}$ :

Structures de surface [L.A]

Structures de surface [L.B]

Structures profondes

un double système de règles de transformation permettant de passer des structures de surface de la langue $\mathbf{A}$ aux structures profondes et de celles-ci aux structures de surface de la langue B. Les deux modèles ne s'excluent pas et tentent seulement de présenter d'une façon schématique les deux aspects complémentaires de l'opération traduisante qui peuvent probablement agir d'une façon concomitante.

Le schéma suivant concrétise cette option double :

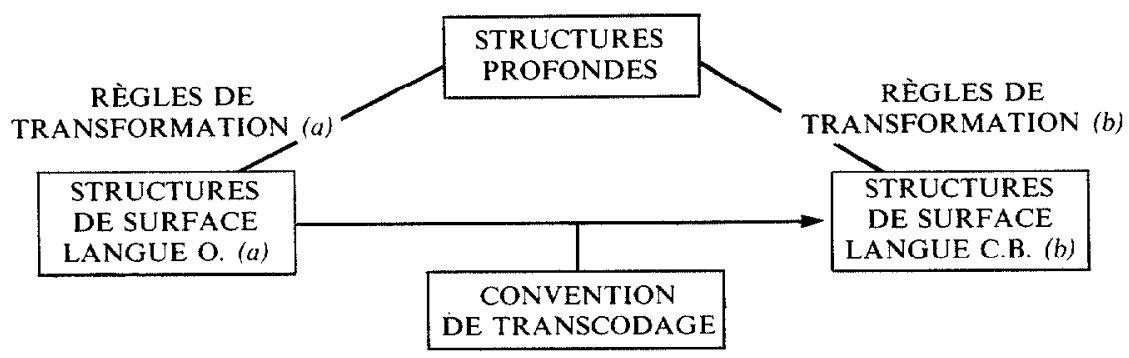

9. Expression et contenu, termes pris dans le sens que leur accorde le linguiste L. Hjelmslev. Tout message comporte à la fois une expression et un contenu, c'est-à-dire qu'il peut ètre envisagé du point de vue du signifiant (expression) ou du signifié (contenu). Voir J. Dubois et al. (1973) : Dictionnaire de linguistique, Paris, Larousse, p. 202.

10. Cf supra. 


\section{LIMITES DES MODẼLES LINGUISTIQUES}

Malgré leur apparente rigueur, les modèles linguistiques que l'on vient de présenter n'intègrent pas tous les paramètres de l'opération traduisante. L'un des obstacles les plus graves auxquels se heurte l'acte de traduire tient aux caractères idiosyncrasiques que chaque langue établit avec la réalité qu'elle doit exprimer au niveau de la convention des signes du langage. S'il y a des universaux sémantiques, proposition que postule justement la théorie générative, ils subissent au niveau des structures de surface particulières à chaque idiome de bien étranges transformations! En l'absence de règles adéquates que la théorie reste jusqu'à présent bien incapable de lui fournir en de nombreux cas, le sujet traducteur doit faire appel à une expérience globale, le savoir dont nous parlions plus haut, qui lui permet de forger, en se fondant sur sa seule intuition, des solutions qui pour aussi empiriques qu'elles soient n'en sont pas moins acceptées comme valables du fait de leur efficacité. Tous les traités de stylistique comparée sont pleins d'exemples de telles trouvailles. Il n'est donc pas utile de nous y attarder ici.

Faut-il en conclure que les modèles linguistiques, tout en donnant de l'opération traduisante des interprétations théoriques satisfaisantes sont incapables d'aider sur le plan pratique l'acte même de la traduction. Ce serait excessif. Ils en légitiment bien des aspects. Mais l'on peut se demander s'il ne conviendrait pas d'élargir le champ même de la théorie pour trouver ailleurs des voies plus fructueuses.

Ainsi, la théorie guillaumienne du langage dans sa double expression de la psychomécanique et de la psychosystématique est peut-être capable de proposer de l'acte de la traduction des analyses linguistiques plus proches de la réalité même des opérations mentales et linguistiques qu'un tel acte suppose. ${ }^{11}$. La réflexion guillaumienne a en effet toujours tenu particulièrement compte du sujet parlant dans son effort pour comprendre les faits du langage. Elle a toujours tenté d'embrasser dans une même observation le mouvement continu qui va de la saisie de la pensée à son investissement en discours alors qu'une approche plus généralement suivie dans le champ de la linguistique postsaussurienne en Europe, postsapirienne en Amérique du Nord, a régulièrement suivi l'orientation contraire.

Or il semble que pour donner de l'opération traduisante une interprétation linguistique satisfaisante il convient à la fois de revenir au sujet qui traduit et à ce mouvement qui va de la pensée au discours. On peut donc espérer que des études de plus en plus nombreuses tenteront d'utiliser les concepts de la psychosystématique et de la psychomécanique pour rendre compte de l'opération traduisante ${ }^{12}$. En ce qui nous concerne ici, cette revalorisation du sujet parlant et des mécanismes qui vont de la pensée au

11. Une bonne introduction aux théories de $\mathrm{G}$. Guillaume se trouve dans louvrage très utile de $R$. Valin (1954) : Petite Introduction à la psychomécanique du langage, Québec, Les Presses de l'Université Laval. On définira : La psychomécanique : La langue est un avant dont le discours forme l'après. L'acte de langage effectue continûment le transfert du premier stade vers le second: son étude ressortit à une linguistique de la pensée pensante: la psychomécanique. La psychosystématique : La langue est un dépôt permanent de structures abstraites à partir desquelles le discours improvise des exploitations momentanées. L'étude des schèmes institués appartient à la psychosystématique.

Ces deux définitions sont empruntées à l'article de M. Wilmet "Psychomécanique et stylistique ", dans l'ouvrage collectif : Langage et psychomécanique du langage pour Roch Valin (1980) : Presses universitaires de Lille, Presses universitaires de Laval, Québec, p. 415.

12. Dans l'ouvrage collectif cité dans la note précédente, il faut lire l'article de G. Garnier : "Psychosystématique et traduction ", pp. 479-494. 
discours suffit amplement à justifier notre démarche qui nous conduit nécessairement à nous tourner vers le sujet traduisant.

\section{DONNÉES NEUROPHYSIOLOGIQUES}

\section{LE SUPPORT SOMATIQUE DU LANGAGE}

Le support somatique du langage ou, pour être plus précis de l'acte individuel de la parole, est postulé aux deux niveaux d'organisation de la langue : le niveau phonologique et morphosyntaxique du plan de l'expression, celui lexico-sémantique du plan du contenu. Certains aspects de ce support somatique au niveau du plan de l'expression relèvent si évidemment du domaine du physique, donc de l'observable, qu'ils s'imposent comme immanents.

Reprenant une longue tradition de réflexion sur cette relation du langage et du corps, Géraud de Cordemoy ${ }^{13}$ réaffirme, à la fin du XVII ${ }^{\mathrm{e}}$ siècle, le double aspect physique et mental de l'acte du langage. Il y voit comme une symétrie en miroir de ce qu'il considère comme la double nature de l'homme. Après lui, il faudra encore presque deux siècles pour que ce " mental "soit finalement ramené à une réalité physique au niveau de l'organisation nerveuse.

Au cours du XVIII ${ }^{\mathrm{e}}$ siècle les connaissances sur la nature de la voix se précisent de Dodard ${ }^{14}$ à Ferrein ${ }^{15}$ et à Vicq d'Azir ${ }^{16}$. Mais comme le note dès la fin du XVII ${ }^{\mathrm{e}}$ siècle B. Lamy ${ }^{17}$, le problème n'est pas de savoir comment, par l'intermédiaire de l'instrument la voix se forme, il convient aussi de comprendre comment cette voix est modulée de telle façon par le sujet parlant qu'elle produit des suites de sons articulés, porteurs de sens et quelle est la nature des mécanismes humains qui accordent à tel type d'articulation une valeur signifiée spécifique.

Avant d'arriver à formuler une théorie de la localisation cérébrale de telles capacités il fallut d'abord acquérir une meilleure connaissance du cerveau lui-même et de la nature des échanges qu'il permet par l'intermédiaire des nerfs à l'intérieur du corps et à l'extérieur de celui-ci avec son environnement.

Du côté de la clinique, les observations se font de plus en plus fréquentes, dans la seconde moitié du XVIII ${ }^{\mathrm{e}}$ siècle, de cas de perte de la parole survenant après des traumatismes crâniens. Durant la même période, des autopsies de plus en plus nombreuses révèlent la probabilité d'une étroite relation entre tel type de lésion corticale et des altérations profondes de la parole.

À partir du début du XIX $\mathrm{X}^{\mathbf{e}}$ siècle, sous l'influence des théories phrénologiques de Gall ${ }^{18}$, on commence à envisager beaucoup plus sérieusement l'éventualité d'une localisation en un espace précis du cerveau de la faculté du langage. En 1825. Bouillaud ${ }^{19}$, se

13. Géraud de Cordemoy (1668) : Discours physique de la parole. Le texte se trouve dans l'édition critique de P. Clair et F. Girbal (1968) : G. De Cordemoy (1626-1684), oeuvres philosophiques, avec une étude bio-bibliographique, Paris, PUF.

14. D. Dodart (1700, compléments $1706 \&$ 1707) : Mémoire sur les causes de la voix de l'homme et de ses différents tons", in : Hist. Acad. roy. des Sci.

15. A. Ferrein (1741) : "De la formation de la voix de l'homme", in : Hist. Acad. roy. des Sci.

16. F. Vicq d'Azyr (1779) : Deux Mémoires sur l'origine de la voix.

17. B. Lamy (1675) : la Rhétorique ou l'art de parler, Paris. Voir L. III, ch. I, p. 188.

N.B. Ce développement résume un chapitre de Ch. P. Bouton (1983) : le Discours physique du langage, contribution à l'histoire des concepts en neurolinguitique, Paris, Klincksieck.

18. F.J. Gall (1825) : Sur les fonctions du cerveau et sur celles de chacune de ses parties, Paris, J.B. Baillière.

19. J.B. Bouillaud (21 février 1825) : Recherches cliniques propres à démontrer que la perte de la parole correspond à la lésion des lobules antérieurs du cerveau et à confirmer l'opinion de $\mathrm{M}$. Gall sur le siège du langage articulé ", Mémoire lu à l'Académie royale de médecine, in : Arch. gles de la méd., année 1825 , t. VIII, pp. $25-45$. 
fondant sur une analyse clinique n'ayant jamais auparavant atteint une telle rigueur et sur des données anatomiques tout aussi rigoureuses, croit pouvoir établir le fait que la faculté du langage articulé est localisée dans ce qu'il nomme les lobules antérieurs du cerveau.

La longue histoire de la recherche d'un lieu du corps pour le langage aboutit ainsi, dans la seconde moitié du XIX $X^{c}$ siècle, sous l'influence de nombreux travaux dont ceux de Broca ${ }^{20}$ à la formation du concept de l'aphasie, nom imposé par Trousseau ${ }^{21}$ à un ensemble de perturbations pathologiques du langage qui commencent à être fort bien reconnues. Le même Broca fonde ce que l'on appellera souvent ensuite le dogme de la localisation dans la troisième circonvolution frontale gauche des fonctions de l'articulation du langage.

Contestée par beaucoup, une localisation aussi stricte fera progressivement place à une systématisation plus complexe des fonctions du langage dans différents lieux du cortex où s'élaborent les perceptions visuelles, auditives et la motricité de la bouche et des doigts, mais la spécialisation de l'hémisphère gauche, considéré comme l'hémisphère du langage, va longtemps être acceptée de presque tous.

Cependant, après la période des théories associationnistes au cours de laquelle on s'efforce de déduire des faits pathologiques des schémas aussi logiques que possible rendant compte de l'organisation du support somatique du langage, la réflexion se dégage, sous l'influence de Freud ${ }^{22}$, de $\mathbf{P}$. Marie ${ }^{23}$ et de Bergson ${ }^{24}$ d'une représentation trop stricte des centres du langage, pour privilégier la notion de fonction. Aux schémas statiques des associationnistes se substituent des diagrammes dynamiques rendant mieux compte de la réalité des processus temporo-spatiaux de l'élaboration de l'acte de la parole.

Le savoir actuel en ce domaine est l'aboutissement de ces démarches dont, au seuil de notre époque, celles de Penfield ${ }^{25}$ et de A.R. Luria ${ }^{26}$, sur la contribution desquels nous reviendrons bientôt.

$\mathrm{Si}$, pour l'essentiel, les fonctions du langage semblent être latéralisées à gauche, soit constitutionnellement, soit d'une manière acquise, l'hémisphère droit, ou d'une manière plus générale, l'hémisphère non dominant, lorsque le droit est dominant, n'est pas pour autant absent de l'élaboration de l'acte du langage, laquelle intéresse par ailleurs des structures profondes du névraxe telles que celles du système limbique ${ }^{27}$ et notamment le thalamus. La pathologie a montré que si l'hémisphère dominant, donc généralement le gauche, se trouve dans l'incapacité de fonctionner, le droit ou l'hémisphère non dominant peut assurer la relève et ceci avec d'autant plus d'efficacité que le trouble s'est 20. Les communications de P.P. Broca figurent de 1861 à 1878 dans le Bulletin de la Société d'anthropologie de Paris et dans le Bulletin mensuel de la Société anatomique de Paris.

21. A. Trousseau (1865) : De l'aphasie, maladie décrite récemment sous le nom impropre d'aphémie ", in : Gazette des hôpitaux, 37 et Clinique médicale. Broca (1864) répondit par une lettre ouverte à Trousseau : "Sur les mots aphémie, aphasie et aphrasie ", lettre à Monsieur le Professeur Trousseau, 18 janvier 1864, in : Gazette des hopitaux civils et militaires, pp. 35-36.

22. S. Freud (1891) : Zur Auffassung der Aphasien, Vienne.

23. P. Marie, sous le titre d'ensemble "Révision de la question de l'aphasie ", fait paraitre dans la Semaine médicale, de 1906 à 1907, cinq articles réunis dans son ouvrage : Travaux et mémoires, paru chez Masson en 1926.

24. H. Bergson (1896) : Matière et mémoire, Paris.

25. L'oeuvre de W.G. Penfield est très importante, pour une sérieuse introduction on consultera Penfield et L. Roberts (1963) : Langage et mécanismes cérébraux, trad. de J.C. Gauthier, Paris, PUF.

26. Les publications de A.R. Luria sont très nombreuses, on consultera d'abord (1976) : Basic Problems of Neurolinguistics, La Haye, Mouton. Cet ouvrage, fondamental, comporte en outre une très complète bibliographie du sujet et particulièrement des oeuvres de A.R. Luria.

27. On entend par système limbique les structures du cerveau connues plus largement sous le terme de rhinencéphale. Il correspond au cerveau paléo-mammifere qui coiffe les structures phylogénétiquement préexistantes, c'est-à-dire le cerveau reptilien. 
manifesté chez des sujets très jeunes tandis que chez l'adulte les récupérations deviennent de plus en plus improbables alors que l'âge s'élève. Le langage implique donc en fait la névraxe dans sa totalité même si des zones fonctionnelles spécifiques ont pu être identifiées. Dans quelle mesure est-il légitime de se demander si la possession de deux ou plusieurs langues modifie l'économie de cette complexe structure d'ensemble?

Il est légitime de poser la question, cela ne signifie pas pour autant que nous avons les éléments de la réponse. Aventurons-nous maintenant sur ce terrain très instable.

\section{DU CER VEAU BILINGUE AU CERVEAU POLYGLOTTE}

De nombreuses hypothèses ont été émises à ce sujet. On peut en dégager les différentes orientations ${ }^{28}$ :

- Chaque langue est emmagasinée dans un lieu différent du cerveau. Cette option qui ne semble pas facilement conciliable avec ce qui précède a eu quelques défenseurs ardents. Elle était encore soutenue en 1959 par Gorlitzer von Mundy ${ }^{29}$. Elle renfermait peut-être, comme on le verra, une étincelle de vérité.

- La possession de deux ou plusieurs langues repose en fait sur les mêmes zones fonctionnelles, mais l'hémisphère non dominant, du fait de la surcharge des circuits neuroniques, est plus impliqué dans l'élaboration des actes de parole chez le bilingue ou le polyglotte que chez le monolingue. C'est un point de vue qu'il est difficile d'attaquer en l'état actuel de nos moyens d'investigation. Certains spécialistes tels que M.L. Albert et L.K. Obler ${ }^{30}$ considèrent très sérieusement l'hypothèse selon laquelle un état de bilingualité entraîne le concours actif de l'hémisphère non dominant. C'est une récupération nuancée de la première hypothèse si unanimement rejetée.

- La deuxième langue, ou les autres langues, se construit sur le même support somatique que la première, mais le jeu concomitant des deux langues suppose une fonction corticale qui permet le passage d'un code à l'autre. Il s'agirait d'une sorte de commutateur linguistique.

À partir de ces trois orientations, toutes les variations sont possibles. En particulier le point de vue qui consiste à dire que la première langue est latéralisée à gauche, tandis que la seconde est soutenue par des fonctions bilatérales.

On se trouve ici dans le domaine de la spéculation pure. La seule réalité sur laquelle l'esprit peut s'appuyer dans cette recherche est représentée par les données de la pathologie. Tournons-nous donc du côté de la clinique.

Depuis la fin du siècle dernier de nombreuses observations de cas d'aphasie chez des bilingues ou des polyglottes ont permis de dégager quelques principes. Pitres ${ }^{31}$ et $\mathrm{Ri}$ bot ${ }^{32}$ croient pouvoir établir le fait qu'après une attaque aphasique, la première langue à revenir est la plus familière, c'est-à-dire le plus souvent la langue maternelle. Cependant de nombreux cas qui paraissent infirmer cette "loi » forcent à ne l'accepter qu'avec

28. Pour avoir une bonne vue d'ensemble de la question, on consultera le travail de M. Paradis dont on utilise ici les données "Bilingualism and Aphasia ", in : H. \& H.A. Whitaker, edits (1977) : Studies in Neurolinguistics, New York, Acad. Press, Vol. III. Une rédaction parallèle, en français, figure dans A.R. Lecours et F. Lhermitte, édit. (1979) : Traité d'aphasiologie, Paris, Flammarion. M. Paradis (1983) vient de publier Readings on Aphasia in Bilinguals and Polyglots, Montréal, Didier.

29. V. Gorlitzer von Mundy (1959) : "Eine 94 Jährige mit einem deutschen sprachzentrum und mit warscheinlich 2 slowenischen Sprachzentren ", in Wiener Medizinische Wochenschrift.

30. M.L. Albert \& L.K. Obler (1978) : The Bilingual Brain, "Perspective in Neurolinguistics", N.Y., Acad. Press.

31. A. Pitres (1895) : "Étude sur l'aphasie chez les polyglottes", in : Revue de médecine, 15, $873-899$.

32. Th. Ribot (1891) : les Maladies de la mémoire, Paris, G. Baillière. 
beaucoup de nuances. En fait l'analyse des processus de récupération montre que la nature des codes linguistiques des langues concernées par le cas joue un rôle considérable dans l'organisation même de la récupération et ceci en fonction de la localisation de la lésion. Ainsi Albert et $\mathrm{Obler}^{33}$ rapportent avoir vu un cas de sujet multilingue souffrant de lésions corticales graves et qui présentait le tableau clinique d'une aphasie de Broca dans une langue et de Wernicke dans l'autre. A.R. Luria ${ }^{34}$ a étudié le cas d'un journaliste français parlant couramment le polonais, l'allemand et le russe. Devenu aphasique à la suite d'un traumatisme crânien, il récupéra lentement l'usage parlé des quatre langues avec quelques déficits dans l'intonation et la mélodie. La récupération de l'écriture présenta un tout autre tableau. En russe, écriture cyrillique, son orthographe était à peu près correcte, en français par contre, écriture latine aux grandes irrégularités phonétiques, ses fautes étaient très sévères. McDonald Critchley note également que chez un Anglo-chinois, bilingue, le modèle aphasique différera selon que les aires auditives ou visuo-kinesthésiques, soit la région temporale ou la région pariéto-occipitale, sont plus ou moins affectées. Une aphasie due à une lésion temporale déterminera une importante dysgraphie en anglais, tandis que l'écriture en chinois sera conservée, l'inverse se produira dans le cas d'une lésion pariéto-occipitale 35

De telles données ne permettent pas de dire que chez un même sujet l'usage de deux ou plusieurs langues repose sur des supports somatiques totalement indépendants, mais elles laissent supposer que si le support somatique est identique, son organisation de langue à langue et ses mécanismes de fonctionnement sont, dans une certaine mesure, différenciables. Sans qu'il soit nécessaire de nous étendre plus sur ces données ${ }^{36}$, nous pouvons revenir à notre sujet, le traducteur.

\section{LE CERVEAU DU TRADUCTEUR}

Le traducteur est un bilingue ou un polyglotte comme les autres, mais c'est un bilingue ou un polyglotte conscient de sa condition linguistique. Alors que le bilingue qui n'est que bilingue est guetté constamment par les pièges de l'interférence ${ }^{37}$, le traducteur même s'il s'y laisse parfois prendre est toujours sur ses gardes. On peut donc supposer que s'il existe bien dans l'organisation corticale une chose telle qu'un commutateur linguistique, ce mécanisme est chez le traducteur particulièrement bien "huilé »!

Cependant cette fonction d'aiguillage que d'aucuns veulent localiser dans le gyrus supramarginalis (zone pariéto-temporale) a-t-elle une représentation somatique aussi spécifique?

Le cerveau du traducteur, selon toutes probabilités doit fonctionner en faisant appel au même support somatique que celui de n'importe quel autre sujet parlant. Il semble raisonnable d'admettre aujourd'hui que du fait de l'héritage génétique avec lequel l'être humain naît, il possède un cerveau qui lui permet à la fois de générer "du langage doublement articulé " et de développer à partir de cette initiale compétence une compétence au second degré pour fonctionner linguistiquement dans la langue de son milieu. L'emploi précoce ou concomitant de deux systèmes linguistiques qui permet une condition satisfaisante de bilingualité 38 individuelle n'est qu'un aspect particulier du phéno-

33. cf. supra.

34. A.R. Luria (1960) : "Differences between Disturbances of Speech and Writing in Russian and French", in : International Journal of Slavic Linguistics and Poetics, 3, pp. 13-22.

35. McDonald Critchley (1974) : Aphasia in Polyglots and Bilinguals ", in : Brain and Language, 1, pp. 15-27.

36. Voir : Ch.P. Bouton, la Neurolinguistique, Paris, PUF, "Que sais-je?", sous presse.

37. A. Martinet (1952) : "Diffusion of Language and Structural Linguistics ", in : Romance Philology, no 1, pp. 5-13.

38. Dans Ch. P. Bouton la Neurolinguistique, nous expliquons que nous proposons d'utiliser le terme de bi- 
mène général de développement verbal de l'enfant. Le traducteur qui peut-être émergera sur cette constitution initiale de bilingualité aura simplement bénéficié des conditions optimales pour y parvenir. Un sujet accédant plus tardivement au statut de bilingue et à la fonction de traducteur n'aura pas, lui, joui des mêmes facilités pour y parvenir. À cet égard, on peut se demander s'il n'existe pas une différence d'organisation neuronale dans le premier cas et dans le second. Par expérience, on doit reconnaître qu'il existe fonctionnellement une différence dans le degré d'automaticité des mécanismes de production de la parole entre une langue acquise au berceau et une autre étudiée bien plus tard. Une telle différence repose fort probablement sur une réalité somatique qui tient au degré d'intégration de tout système de conduite acquis, soit un apprentissage en termes clairs.

Si le cerveau du traducteur reste comparable à celui de n'importe quel autre sujet parlant, on peut supposer par contre que c'est un cerveau mieux exploité, au rendement évidemment plus élevé, non seulement en ce qui concerne ses performances proprement linguistiques mais dans la totalité de son activité. Ainsi que nous l'avons vu, l'opération traduisante implique à côté d'un "savoir-faire "d'ordre technique, un savoir tout court qui engage la totalité de l'individu et donc la totalité de l'activité corticale. Trois dimensions de la réflexion neurolinguistique nous permettront de préciser ce qui précède.

\section{TROIS PROPOSITIONS FINALES}

\section{LE CORTEX INTERPRÉTATIF}

Au cours des exérèses corticales dont il avait mis au point la technique opératoire, Penfield nota régulièrement le phénomène suivant ${ }^{39}$ : quand la stimulation électrique touche certains points du lobe temporal, on obtient du patient des réponses psychiques qui peuvent être divisées en deux groupes, réactions vécues, réactions interprétatives. La réaction vécue est une sorte de fulgurant retour en arrière. Un souvenir oublié réapparaît avec une étonnante précision, que le patient verbalise aussitôt : «J'étais là, quelqu'un m'appelait ... un homme penché sur un pupitre avec un crayon à la main... ". La réaction interprétative se rapporte au présent vécu par le patient, mais comme "distancié » par rapport à ce présent. Ce qui se passe autour du patient prend une sorte de recul comme s'il s'agissait d'un souvenir réactualisé ${ }^{40}$.

Ces observations conduisirent Penfield à supposer l'existence d'un cortex interprétatif s'ajoutant au cortex sensoriel et au cortex moteur. Selon son hypothèse, il s'agirait d'un lieu du cerveau où le " fil du temps " de chacun viendrait s'imprimer. Chaque individu, précise-t-il, constitue un enregistrement neuronal de son propre courant de conscience. La stimulation électrique ne fait que raviver ces engrammes du souvenir. Derrière la métaphore du livre, du disque ou de la bande magnétique, selon la technique prise comme référence, le fait clinique subsiste. Stimulé par l'effort de la concentration, c'est à ce réservoir de souvenirs que notre savoir verbal s'alimente, se façonne et devient porteur de sens. Quand un sujet possède deux codes linguistiques, c'est à cette source commune qu'ils doivent obligatoirement se revitaliser à chaque acte de parole.

Pour le traducteur, la même banque d'informations constituée au cours de son temps, nourrie par son expérience des choses et des êtres est donc constamment réinves-

lingualité, pour distinguer le bilinguisme de la personne, condition de bilingualité, du bilinguisme institutionnel, celui d'un état, par exemple. La bilingualité pourrait donc être définie comme un aspect particulier de la condition plus générale de polyglossie ou polyglottisme.

39. W.G. Penfield (1975); The Mystery of Mind, Princeton, N.J. Princeton Univ. Press. On peut aussi con. sulter : Langage et mécanismes cérébraux.

40. Voir Langage et mécanismes cérébraux, particulièrement pp. 41-55. 
tie lors de chaque acte de traduction. Mais, s'il possède des deux langues, véhicules de cultures et de civilisations distinctes dans le sens le plus large que l'on peut donner à ces termes ambigus, une expérience vivante, le cortex interprétatif sur lequel il s'appuie lui fournit immédiatement le système d'équivalences qu'aucune théorie linguistique du sens ne peut actuellement lui proposer. C'est ainsi qu'il peut surmonter le handicap de la traduction littérale, génératrice de contre-sens sinon de faux-sens !

La proposition suivante vient en quelque sorte renforcer la précédente alors qu'elle dérive d'une orientation différente de la réflexion.

\section{LE MOT, MATRICE MULTIDIMENSIONNELLE}

Le signe linguistique, avait constaté F. de Saussure, est doublement motivé : morphologiquement et sémantiquement. Cette double motivation est évidente lorsque l'on constate chez chaque sujet parlant la double disponibilité de chaînes associatives tant formelles que sémantiques. A.R. Luria ${ }^{41}$ et son équipe ont montré que cette réalité linguistique reposait sur des systèmes de structures neuroniques différents du fait des réactions conditionnelles qu'il est possible de faire acquérir à l'organisme à partir de ces phénomènes associatifs mêmes 42

Mais, au-delà de cette double chaîne associative que contrôle l'expérimentation, chaque mot, ou plutôt chaque groupe minimal d'unités linguistiques porteur de sens rassemble sur le support matériel de la séquence sonore que ce groupe représente sur le plan de l'expression, l'ensemble des expériences cognitives ou sensorielles postulées par le sens, sur le plan du contenu.

On peut entrevoir la complexité de l'organisation somatique sur laquelle repose en fait l'émergence du sens ${ }^{43}$ Prenons par exemple des termes concrets tels que orange ou pomme. Selon ce qui précède, ils ont d'abord une réalité somatique à un double niveau :

- Des ensembles praxiques, combinatoires de connexions déterminant les gestes articulatoires qui permettent la production de la suite de sons constituant, sur le plan de l'expression, la matérialité phonique du mot.

- Des ensembles gnosiques permettant à la fois l'identification du message auditif et donc la reconnaissance dans la suite de la chaîne sonore continue de l'énoncé, de l'identité de chacun des mots qui le constituent.

Il faut tenir compte du fait que ces deux systèmes praxiques et gnosiques se dédoublent par l'apprentissage de l'écriture et de la lecture, aboutissant à constituer un double soussystème praxique et gnosique étroitement associé au précédent et en quelque sorte complémentaire.

Ces ensembles et sous-ensembles sont étroitement interconnectés de telle sorte qu'ils ont la propriété de se stimuler réciproquement.

Mais, chacun des termes précités, orange ou pomme, s'associe, sur le plan perceptif, dans sa matérialité somatique à la fois praxique et gnosique, à des faisceaux d'informations non linguistiques qui tiennent aux données sensorielles se déduisant de l'expérience de la chose elle-même. Ainsi, l'odeur ou la couleur font surgir dans le champ de la conscience le terme orange ou le terme pomme. C'est par le jeu de ces stimulations praxiques ou gnosiques réciproques que se dessinent des configurations sensitives ou cognitives d'où surgit, sur le plan verbal, l'image : métaphore ou métonymie.

Du fait de la nature particulière de la relation ainsi établie entre le mot dans sa réalité linguistique et le système de références praxiques et gnosiques qu'il postule au ni-

\footnotetext{
41. A.R. Luria (1974) : "Basic Problems of Neurolinguistics", in : Current Trends in Linguistics, Th. Sebeok, Ed., La Haye, Mouton, vol. 12****, pp. 2561-2594.

42. Voir le texte de Luria cité dans la note précédente, p. 2575 sqq.

43. Présentation reprise de Ch. P. Bouton la Signification, p. 295 sqq
} 
veau de la réalité somatique, chaque terme d'une langue donnée représente, pour reprendre le terme de Luria, une matrice spécifique avec ses propres systèmes de relations associatives. Ce sont probablement de tels systèmes qui permettent chez le bilingue averti qu'est le traducteur, de "sentir ", par intuition les aires d'extension ou de limitation sémantiques propres à chacun des ensembles lexicaux qu'il exploite et met en relation de langue à langue.

Ainsi, le terme russe Kraniy est bien l'équivalent du terme français rouge quand il s'agit de se reférer à la couleur. Toutefois, le terme russe suggère également le sens métaphorique de [beau], ce qui n'est pas possible en français.

Il est tentant, à titre d'hypothèse, de suggérer par conséquent que le cerveau bilingue présente un double système d'interconnections et que chacun de ces systèmes est d'une façon ou d'une autre également interconnecté. Le caractère volontairement vague de la formulation en souligne la valeur strictement heuristique. C'est avec la même prudence qu'il convient d'introduire la troisième proposition.

\section{LA FONCTION RÉGULATRICE DU LANGAGE}

Les psychologues ont constaté depuis longtemps la grande différence existant entre les conduites de l'enfant, avant le langage et après son acquisition. Dans l'évolution de ces conduites, le développement cognitif général de l'enfant joue inconstestablement un rôle essentiel, mais celui-ci ne diminue pas pour autant le rôle même du langage. Le langage permet au sujet de se "programmer " avant l'action et de se contrôler durant. D'une manière plus ambitieuse, sans tomber dans le piège d'une spéculation philosophique que nous voulons éviter ici, disons que par le langage l'homme se pense à la fois comme être pensant, sentant et agissant.

Les données de la pathologie renforcent ce point de vue théorique. Depuis plus d'un siècle le problème de la relation entre l'aphasie et la perte de l'intelligence est posé en termes nets. Il n'est pas pour autant résolu ${ }^{44}$. Ce que l'on peut prudemment avancer, c'est que selon les formes de la lésion et la nature du déficit verbal, les conduites du malade sont plus ou moins altérées, mais en un certain sens elles le sont toujours. Les troubles de cette fonction supérieure du langage sont encore plus manifestes dans certains types de démences ${ }^{45}$. Les conduites du sujet sont altérées selon les perturbations dans la relation qu'il entretient avec lui-même, dans la relation qu'il entretient avec les autres ou dans les relations qu'il entretient, dans un sens plus général, avec la réalité.

A.R. Luria, encore lui !, a nettement postulé l'existence de l'organisation somatique d'une telle fonction régulatrice assumée par le langage ${ }^{46}$. Sans aller jusqu'à la vision dramatique d'un éclatement de la personnalité de forme schizoïde, il est juste de se demander dans quelle mesure la totale maitrise de deux codes linguistiques ne favorise pas une certaine ambivalence de la personnalité. Les fonctions nerveuses supérieures soumises à la censure auto-régulatrice du langage, contrôlées dans ce cas par un double système auto-régulateur pourraient être capables de deux types de conduites selon que l'une ou l'autre langue domine pour un temps l'ensemble de l'activité nerveuse supérieure. Dans quelle mesure, alors, la situation même du sujet au cours de l'opération tra-

44. Consulter Le discours physique du langage et notamment le chapitre IV-I-2, " $\dot{A}$ la recherche de l'aphasie".

45. Consulter L. Irigaray (1973) : le Langage des déments, La Haye, Mouton. Voir aussi l'ouvrage de G. Lantéri-Laura (1966) : les Apports de la linguistique à la psychiatrie contemporaine, Paris, Masson. Voir aussi T. Todorov, "Le discours psychotique", in : P. Léon et H. Mitterand (1979) : l'Analyse du discours, Montréal, Centre éducatif et culturel, pp. 49-60.

46. A.R. Luria (1961): The Role of Speech in Regulation of Normal and Abnormal Behaviour, London, Pergamon Press. 
duisante ne serait-elle pas une situation de crise ou tout au moins génératrice de moments critiques? L'expérience de chacun peut dans une certaine mesure apporter une réponse à cette interrogation.

\section{POUR CONCLURE}

Un double système de décodage de l'enregistrement neuronal du cortex interprétatif, un double système de circuits associatifs au niveau du mot considéré comme matrice multidimensionnelle, un double système auto-régulateur des conduites et des comportements, les uns et les autres mis en relation par des "ponts " permettant le passage d'un code linguistique à l'autre, le cerveau du traducteur, s'il n'est pas réellement organisé différemment de tout autre, est tout au moins un cerveau équipé pour fonctionner avec un double rendement. Pour reprendre la métaphore chère au XVIII mettant au goût de notre temps, c'est une machine à double commande.

Limitons cependant la portée de nos propos. S'ils se basent sur des données authentiques, ils les ont dépassées pour se situer au niveau de la pure spéculation. Ce n'est pourtant pas un simple jeu d'esprit. C'est une procédure de découverte! 